\title{
Microstructure and Characterization of CuAINi Shape Memory Thin Films
}

\author{
A.C. Kneissl, K. Kutschej, X.D. Wu \\ University of Leoben, Franz-Josef-Strasse 18, 8700 Leoben, Austria
}

The shape memory effect (SME) occurs in many alloy systems, e.g. NiTi, CuZnAl and CuAlNi. The effect is characterized by a reversible shape change which is caused by temperature changes (oneway effect, two-way effect) or external stresses (pseudoelasticity). These alloys are used to an increasing degree for industrial applications. The efforts aim at a miniaturization of the components to use them as integrated parts of so called intelligent structures. For this reason attempts were made to produce thin films by PVD coating with an alloy $\mathrm{Cu}-25.8 \mathrm{Al}-3.6 \mathrm{Ni}$ at\%, which shows the characteristic properties of a temperature controlled shape memory material (up to $250^{\circ} \mathrm{C}$ ) because of the phenomenon of thermoelastic martensitic transformation [1]. The deposition took place on base of magnetron sputtering, using a PVD plant from Leybold-Heraeus. The vacuum value of the chamber was $10^{-3} \mathrm{~Pa}$, Ar-gas was admitted up to a working pressure of 0.4-3.8 Pa. Glass was used as the substrate material. Different variations of the sputter parameters (Ar-gas working pressure, current, substrate temperature, time) were tested in order to receive a removable, high quality thin film with good shape memory properties. A correlation between the sputter parameters and the quality of the film was to be established. Ar-gas working pressures exceeding $2 \mathrm{~Pa}$ and substrate temperatures over $100^{\circ} \mathrm{C}$ had a negative influence on the removability. At pressures between 0.4 and $2 \mathrm{~Pa}$ good, slightly removable films could be produced. Therefore the following parameters were used: 0.4-2 Pa Ar-gas working pressure, 1-1.5 A electrical current and a maximum temperature of $90{ }^{\circ} \mathrm{C}$ for the substrate. The films were removed from the glass substrate with the aid of a scalpel.

The composition of the material has an important influence on the desired shape memory properties [2]. Therefore the films were analysed by means of EDX. It was found in our work that the aluminium concentration of the film was always smaller than the value of the aluminium concentration of the target caused by the lower sputter rate of $\mathrm{Al}$ and stronger pushing and scattering effects of the lighter Al-Atoms compared to the $\mathrm{Cu}$-Atoms [3]. The Ar-gas working pressure shows the biggest influence on the $\mathrm{Al}$ and $\mathrm{Cu}$ concentration. With increasing pressure the $\mathrm{Al}$ content decreases and the $\mathrm{Cu}$ content increases while the $\mathrm{Ni}$ content stays approximately constant. After these experiments we are able now to predict fairly good the composition of a film depending on the sputter parameters. It is known [4] that at low and medium cooling rates the body-centred hightemperature phase $\beta$ decomposes into the equilibrium phases ( $\alpha$ and $\left.\gamma_{2}\right)$. This can occur also during PVD processes. To secure the transformation into martensite annealing of the films $\left(800^{\circ} \mathrm{C} / 60\right.$ minutes) with subsequent quenching had to be done [5]. To characterize the morphology and the microstructure, REM and TEM investigations were performed. Figure 1 shows the fracture surface of thin films with and without heat treatment. The layer thickness at a sputter time of 45 minutes is approximately $7-10 \mu \mathrm{m}$. The fracture surface in Figure 1a shows a porous, irregular structure. The heat treated film on the other hand displays a regular, dense structure without pores (Figure 1b) and an average grain size between $0.5-2 \mu \mathrm{m}$. Therefore microstructure had to be investigated with TEM. Specimen with a diameter of $3 \mathrm{~mm}$ were thinned electrolytically and analyzed with a TEM Philips CM12 at $120 \mathrm{kV}$. The martensitic structure is shown in Figure 2. DSC measurements were carried out using a low-temperature DSC instrument from Mettler Toledo Star System. There is a continuous shape of the curves without a phase transformation. If a phase change occurs a peak will 
be shown. From this the martensite- and the austenite-start/finish-temperatures could be deduced. Figure 3 shows the DSC curves of a heat treated specimen. To verify the different phases of the films, XRD measurements were made. Figure 4 shows the XRD spectra of the same sample as in Figure 3. Most peaks could be identified as martensitic phase peaks $\left(\beta_{1}{ }^{\prime}\right)$, only some may be equilibrium phase peaks $(\alpha)$. It also was shown that after manual bending and heating to $180^{\circ} \mathrm{C}$ of these films the original shape was recovered, and thus the one-way SME could be demonstrated. Results will be shown and discussed in detail during the presentation of the paper. [6]

\section{References}

[1] T.W. Duerig, J. Albert, G.H. Gessinger, Journal of Metals 35/12 (1982) 14-20

[2] S.W. Husain, P.C. Clapp, Journal of Materials Science 22 (1978) 509-516

[3] R. A. Haefer, Oberflächen und Dünnschicht Technologie, Teil 1, Verlag: Springer (1987)

[4] S. Miyazaki, K. Otsuka, ISIJ International 29 (1989) 353-377

[5] H. Scherngell, A. Schnabl, A.C. Kneissl, Praktische Metallographie 30 (1999) 375-382

[6] Financial support by the Austrian Science Foundation (FWF project No:14221) is gratefully acknowledged.

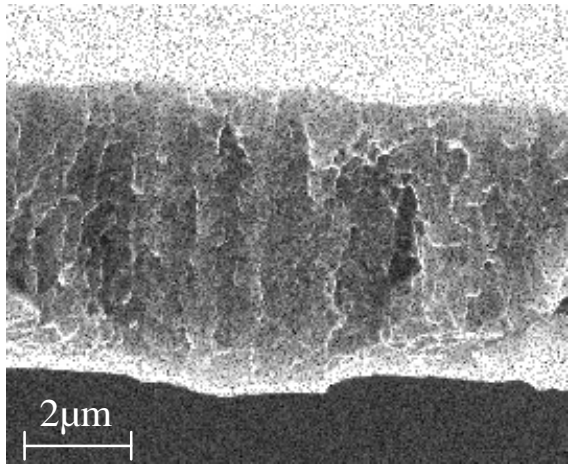

a.) without heat treatment

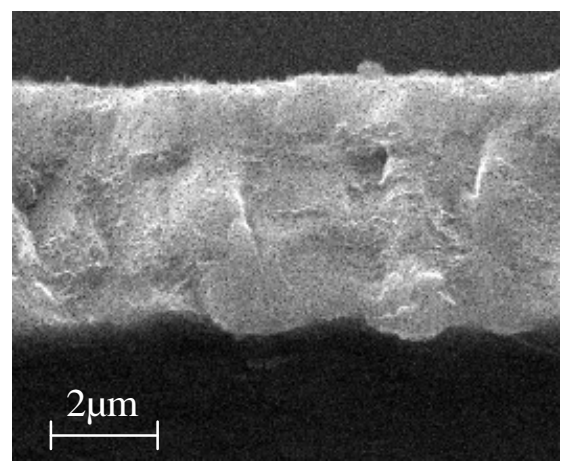

b.) heat treated at $800^{\circ} \mathrm{C} / 60 \mathrm{~min}$

Figure 1 : scanning electron microscopy - fracture surface

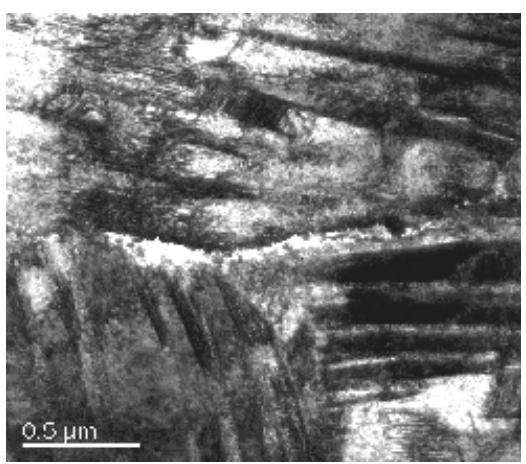

Figure 2 : TEM, heat treated

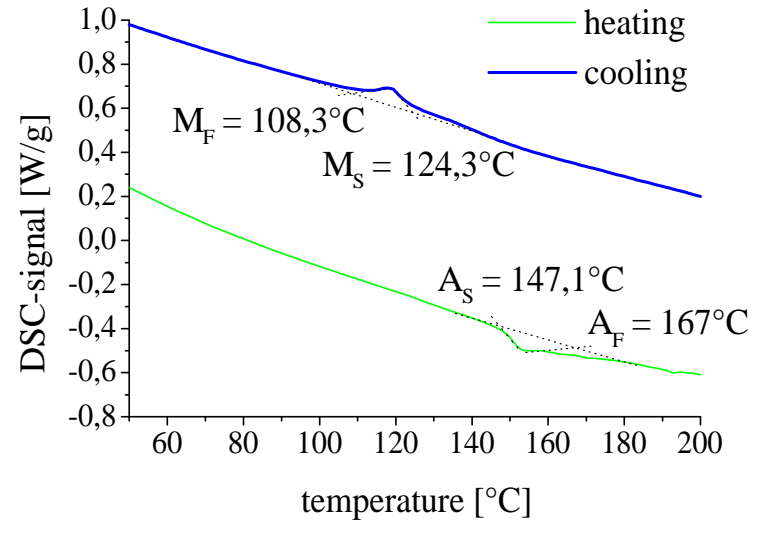

Figure 3 : DSC, heat treated film

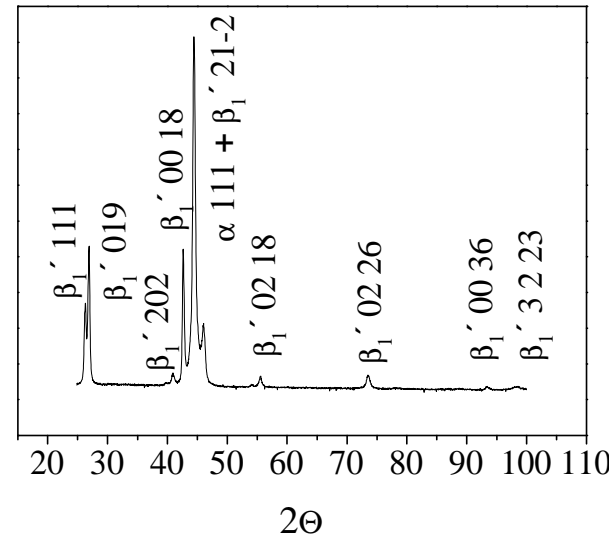

Figure 4 : XRD, heat treated film 